\title{
Resolving the Structure of $\mathrm{Pt} / \mathrm{Mo}_{2} \mathrm{C}$ Catalysts on MWCNTs Using Aberration Corrected STEM
}

\author{
Cem Akatay ${ }^{1}$, Kaiwalya Sabnis ${ }^{2}$, Fabio Ribeiro ${ }^{2}$ and Eric Stach $^{3}$
}

1. School of Materials Engineering and Birck Nanotechnology Center, Purdue University, West

Lafayette, Indiana 47907, USA

2. Forney Hall of Chemical Engineering, Purdue University, West Lafayette, Indiana, USA

3. Center for Functional Nanomaterials, Brookhaven National Laboratory, Upton, New York 11973, USA

Pt supported molybdenum carbide catalysts $\left(\mathrm{Pt} / \mathrm{Mo}_{2} \mathrm{C}\right)$ are reported to have higher rates for water-gas shift reaction when compared to metal oxide supported catalysts [1]. Bulk $\mathrm{Mo}_{2} \mathrm{C}$ support particles are porous in nature and provide poor contrast for HAADF-STEM to identify the entirety of Pt species for statistical analysis. Further, the X-ray absorption spectroscopy (XAS) method is a bulk sensitive technique and thus the signal resulting from the Mo in close contact with $\mathrm{Pt}$, is swamped by the Mosignal originating from the support itself. To take full advantage of both techniques (STEM, XAS), we have synthesized the $\mathrm{Pt} / \mathrm{Mo}_{2} \mathrm{C}$ catalysts on multiwall carbon nanotubes (MWCNT). Carbon nanotube support provides nice contrast to detect Mo and Pt clearly, further eliminating the bulk Mo support enhances the proportion of the Mo XAS signal that is relevant to our studies. Extensive kinetic testing of both bulk $\mathrm{Pt} / \mathrm{Mo}_{2} \mathrm{C}$ and $\mathrm{Pt} / \mathrm{Mo}_{2} \mathrm{C} / \mathrm{MWCNT}$ catalysts are performed to ensure that the reaction is catalyzed in the same manner in both systems.

A series of 10 wt. $\% \mathrm{Mo}_{2} \mathrm{C} / \mathrm{MWCNT}$ with varying Pt loading $(0,0.5,3$, and 5 wt. \%) are tested kinetically and characterized by XAS and STEM. Figure 1a exhibits a HAADF-STEM image of the 5 wt. \% Pt/10 wt.\% $\mathrm{Mo}_{2} \mathrm{C} / \mathrm{MWCNT}$ catalysts. A magnified image of carbon nanotube shows patches of $\mathrm{Mo}_{2} \mathrm{C}$ along with bright Pt particles on top (Figure 1b). The elemental assignments of the regions are confirmed by electron energy loss spectroscopy (EELS). The $\mathrm{Mo}_{2} \mathrm{C}$ and $\mathrm{Pt}$ regions are quantified by area as shown in Figure 1c. STEM-EELS elemental line-scans are performed and the particles are predominantly found to be well-mixed Pt-Mo alloys as shown in Figure 2. STEM intensity quantification is performed to identify the structure and a model is shown in Figure 3. Based on these results, the structure of the $\mathrm{Pt} / \mathrm{Mo}_{2} \mathrm{C} / \mathrm{MWCNT}$ is resolved and WGS rate seems to correlate with Pt-Mo alloyed particle area.

We acknowledge support from the Institute for Atom-efficient Chemical Transformations (IACT), an Energy Frontier Research Center funded by the U.S. Department of Energy, Office of Science, Office of Basic Energy Sciences. E.S. acknowledges additional support from the Center for Functional Nanomaterials, Brookhaven National Laboratory, which is supported by the U.S. Department of Energy, Office of Basic Energy Sciences, under Contract No. DE-AC02-98CH10886.

Research carried out in part at the Center for Functional Nanomaterials, Brookhaven National Contract No. DE-AC02-98CH10886

[1] Schweitzer et al, Journal of American Chemical Society (2011), p. 2378-2381 

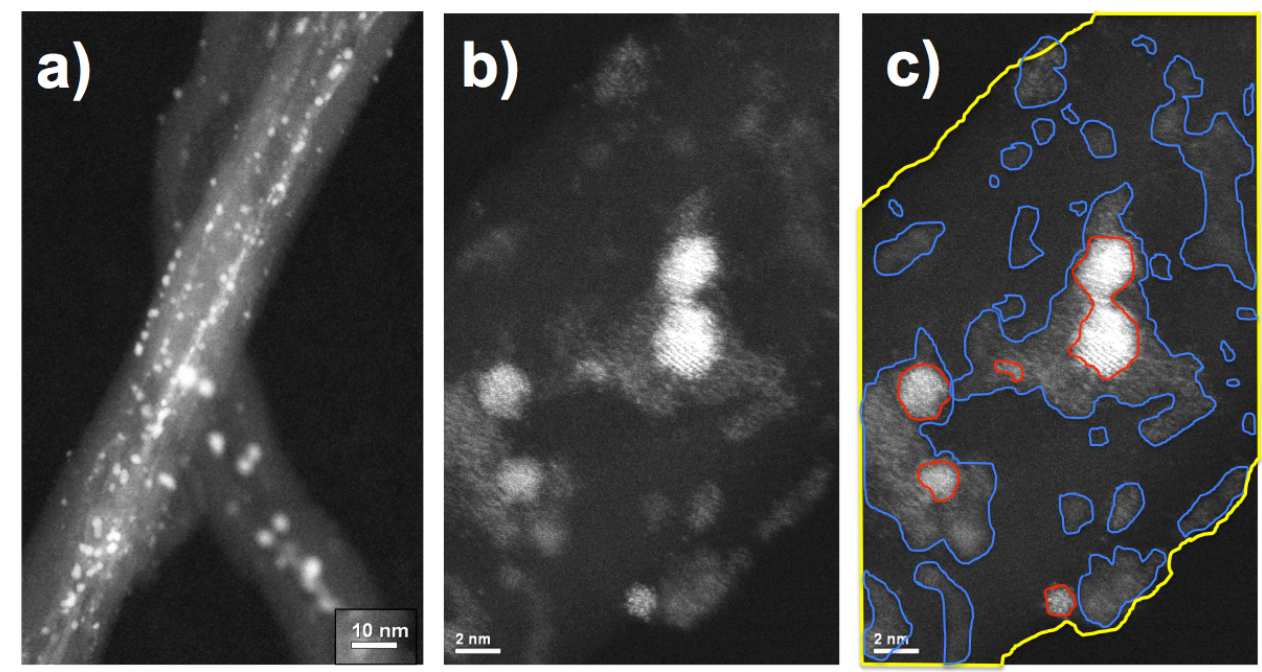

Figure 1. HAADF-STEM micrographs of the 5 wt. \% Pt/10 wt.\% Mo $2 \mathrm{C} / \mathrm{MWCNT}$ catalyst. Pt and Mo area are quantified as shown in c)
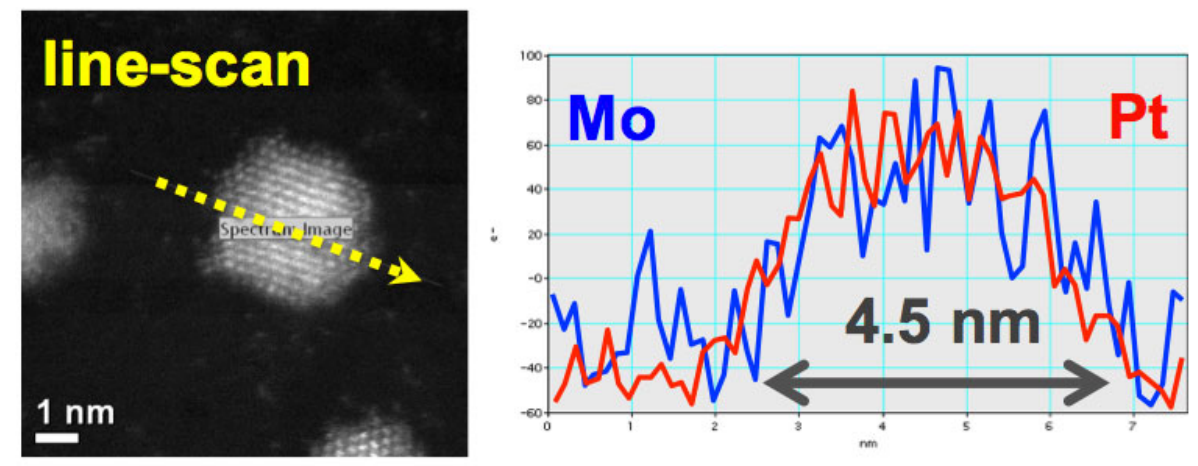

Figure 2. STEM-EELS line-scan on a particle exhibiting a well-mixed Pt-Mo alloy structure

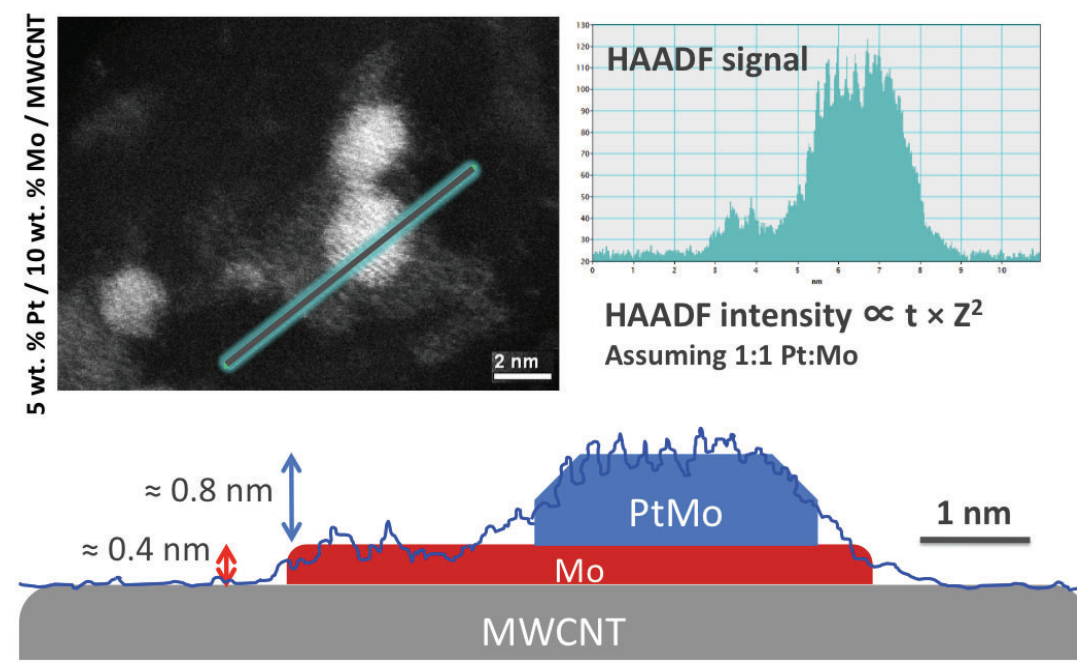

Figure 3. Model of the catalyst structure is developed based on the STEM signal intensity quantification 